\title{
A Reformulation of the Hoop Conjecture
}

\author{
José M. M. SenoviLla ${ }^{1}$ \\ 1 Física Teórica, Universidad del País Vasco, Apartado 644, 48080 Bilbao, Spain
}

PACS 04.70.Bw - Classical black holes

PACS 04.50.Gh - Higher-dimensional black holes, black strings, and related objects

\begin{abstract}
A reformulation of the Hoop Conjecture based on the concept of trapped circle is presented. The problems of severe compactness in every spatial direction, and of how to superpose the hoops with the surface of the black hole, are resolved. A new conjecture concerning "peeling" properties of dynamical/trapping horizons is propounded. A novel geometric Hoop inequality is put forward. The possibility of carrying over the results to arbitrary dimension is discussed.
\end{abstract}

Among the various - yet unproven - beliefs concerning the physics of black holes, a very popular one is the Hoop Conjecture formulated, in a deliberately vague manner, as [49,65]: "black holes with horizons form when, and only when, a mass $M$ gets compacted into a region whose circumference in every direction is $C \lesssim 4 \pi G M / c^{2}$."

The impreciseness of this statement was remarked at ' its birth $[49,65]$, and has been discussed many times, e.g. $[26,57,67]$, for many physical and mathematical difficulties arise. The main problems can be summarized as:

1. In practice, it is impossible to determine the existence of an event horizon, a global concept accessible only to omniscient observers. A remedy has been the use of alternative, local, definitions of horizons, mainly apparent horizons [32,43], based on the concept of closed trapped surface first introduced in [53]. Nowadays, the newer notions of dynamical/trapping horizons $[3,34]$ seem to be a reasonable alternative. One therefore deals with the more fundamental notion of (a world tube of) closed marginally trapped surfaces. I will follow this strategy here.

2 . The use of the symbol $\lesssim$ showing the uncertainty about the numeric constants to be used - several possibilities have appeared in the literature, e.g. [12,13]. It has been even argued that different values should be used for the "when" and the "only when" parts of the conjecture [26].

3. In general, the "circumference" of a bounded body is not defined. In fact, one can pass arbitrarily small hoops around any concentration of matter by letting appropriate portions of the hoop move relativistically (close to a null curve) [67]. A proposed solution in this case is to use a specific slice, usually a particular type of Cauchy hypersurface, e.g. [21,26,46,50,63]. See in this respect, however, the illustrative example in [68], also [52].
4. Another major problem is the definition of mass $M$. Gravitational energy is non-localizable and one has to take into account gravitational radiation. More problematic, even after fixing a slice, is the idea of "mass encircled by the hoop", without solution already in Newtonian physics. The objection here is that there is no notion of region encircled by a hoop (think of a disk or a hemisphere with identical circle as boundary)

Yet, despite all difficulties, the Hoop Conjecture has been successful. It was settled in spherical symmetry [9-11], and discussed in some special non-spherical cases [46]. Many numerical and/or analytical idealized examples $[7,8,12,13,18-21,26,39,50,52,57,63,66,70,71,73]$ have given it robust support. These works (and their references) have sometimes simultaneously considered the cosmic censorship hypothesis $[45,54,67]$, sending conflicting signals, but I will not treat this here. Also, a mathematical result was found in [59] where an upper bound for a "radius" of bounded matter was linked to lower bounds for its mass density. Unfortunately, the size measure in [59] seems to be inadequate, and their criteria rarely met, to describe the extent of black holes - see examples in [10].

Thus, it would be interesting to have a precise formulation, mathematically sound and physically falsifiable, while keeping the spirit of the original conjecture. Its main underlying idea is that black holes must be localized, so that matter-energy must be severely compacted in all spatial directions if horizons are to be formed. But, what is the exact meaning of "severe compactness"? Can this crucial physical idea be formulated in a mathematically correct way? For instance, there is no compelling reason to believe that very elongated matter (such as a long cylinder with two hemispherical caps) cannot collapse to eventually 
form a black hole whenever the height of the cylinder is small enough - compared to the enclosed mass - to produce that "severe concentration". In a way, the concept of closed trapped surface $[32,53]$ captures the idea of severe concentration without speaking of mass, leading to a "compactness conjecture" $[46,60]$. This is related to isoperimetric inequalities $[28,55]$. Other type of inequalities, guaranteeing the existence of averaged closed trapped surfaces, were found in $[46,47]$ in some special situations.

Actually, by means of the mean curvature vector $[42,48$, 51], trapped submanifolds of arbitrary co-dimension can be defined rigorously [48]. This is the new ingredient I want to bring in, because the concept of trapped hoop can thus be used without speaking of its length or its encircled mass. Thus, one can pass the hoops through the designated region and check whether or not they are trapped when instantaneously superposed at relative rest with the limiting surface - avoiding also any slicing.

This concept of trapped hoop allows for a sensible reformulation of the conjecture. More importantly, some reformulated statements can be proven if one is willing to enforce stringent conditions on the closed trapped surfaces. The required stricter types of trapped surface are known as totally, (very) strongly, or (very) lightly trapped surface [62], and are characterized by some properties of their future null second fundamental forms 1$]$

Let $(\mathcal{V}, g)$ be a 4 -dimensional spacetime with metric tensor $g$ of signature $(-,+,+,+)$. Consider an imbedded connected spacelike surface $S[32,51]$, so that its first fundamental form $\gamma$ is positive-definite. Then, at any $x \in S$ the tangent space has the orthogonal decomposition $T_{x} \mathcal{V}=T_{x} S \oplus T_{x} S^{\perp}$ into tangent and normal parts. Using this, the shape tensor of $S$ in $\mathcal{V}$ is defined as

$$
\overrightarrow{\boldsymbol{K}}: \mathfrak{X}(S) \times \mathfrak{X}(S) \longrightarrow \mathfrak{X}(S)^{\perp}, \quad-\overrightarrow{\boldsymbol{K}}(\vec{X}, \vec{Y}) \equiv\left(\nabla_{\vec{X}} \vec{Y}\right)^{\perp}
$$

where $\vec{X}, \vec{Y} \in \mathfrak{X}(S)$ are smooth vector fields tangent to $S$.

Given any normal direction $\vec{n} \in \mathfrak{X}(S)^{\perp}$, the second fundamental form $\boldsymbol{K}_{\vec{n}}$ of $S$ in $(\mathcal{V}, g)$ relative to $\vec{n}$ is the 2covariant symmetric tensor field on $S$ defined by

$$
\boldsymbol{K}_{\vec{n}}(\vec{X}, \vec{Y}) \equiv g(\vec{n}, \overrightarrow{\boldsymbol{K}}(\vec{X}, \vec{Y})), \quad \forall \vec{X}, \vec{Y} \in \mathfrak{X}(S) .
$$

$S$ has two independent normal vector fields, and thus they can be chosen to be null and future-pointing. Call them $\vec{\ell}, \vec{k} \in \mathfrak{X}(S)^{\perp}$, and add the normalization condition $g(\vec{\ell}, \vec{k})=-1$. There remains the freedom

$$
\vec{\ell} \longrightarrow \vec{\ell}^{\prime}=\sigma^{2} \vec{\ell}, \quad \vec{k} \longrightarrow \vec{k}^{\prime}=\sigma^{-2} \vec{k}
$$

where $\sigma^{2}>0$ is a function on $S$. The shape tensor decomposes as $\overrightarrow{\boldsymbol{K}}=-\boldsymbol{K}_{\vec{k}} \vec{\ell}-\boldsymbol{K}_{\vec{\ell}} \vec{k}$ where $\boldsymbol{K}_{\vec{k}}, \boldsymbol{K}_{\vec{\ell}}$ are the future null second fundamental forms.

The mean curvature vector $\vec{H}$ of $S$ in $(\mathcal{V}, g)$ is an averaged version of the shape tensor defined by $\vec{H} \equiv \operatorname{tr} \overrightarrow{\boldsymbol{K}}$,

\footnotetext{
${ }^{1}$ Totally trapped surfaces were called strongly trapped in [44]. For strongly marginally trapped surfaces the two nomenclatures agree.
}

$\vec{H} \in \mathfrak{X}(S)^{\perp}$, where the trace tr is taken with respect to $\gamma$. The scalars $g(\vec{H}, \vec{n})=\operatorname{tr} \boldsymbol{K}_{\vec{n}}$ are termed "expansion along $\vec{n}$ " of $S$. In particular, $\theta_{\vec{k}} \equiv \operatorname{tr} \boldsymbol{K}_{\vec{k}}$ and $\theta_{\vec{\ell}} \equiv \operatorname{tr} \boldsymbol{K}_{\vec{\ell}}$ are called the future null expansions and $\vec{H}$ becomes

$$
\vec{H}=-\theta_{\vec{k}} \vec{\ell}-\theta_{\vec{\ell}} \vec{k} .
$$

$S$ is said to be (semi-) contracting at $x \in S$ if $\left.\vec{H}\right|_{x}$ is (nonzero null) timelike and future-pointing [62]. If these properties are kept on the whole $S$ then $S$ is called (marginally) future trapped. Obviously, $S$ is future trapped if and only if $\theta_{\vec{k}}<0$ and $\theta_{\vec{\ell}}<0$, and marginally future trapped if one of them vanishes and the other is negative, on $S$. Physically, contracting points have local neighborhoods with initially decreasing area along any future direction.

One can also define the vector density [62]

$$
\vec{G} \equiv-\left(\operatorname{det} \boldsymbol{K}_{\vec{k}}\right) \vec{\ell}-\left(\operatorname{det} \boldsymbol{K}_{\vec{\ell}}\right) \vec{k}, \quad \vec{G} \in \mathfrak{X}(S)^{\perp} .
$$

(2) is invariant under (11), but not (3). However, the causal orientation of $\vec{G}$ is invariant under (11). The combination of $\vec{H}$ and $\vec{G}$ provides a refined classification of trapped surfaces [62], in particular a future trapped $S$ is called:

- totally future trapped if $\vec{G}$ is past-pointing timelike on $S$; equivalently, both $\operatorname{det} \boldsymbol{K}_{\vec{k}}$ and $\operatorname{det} \boldsymbol{K}_{\vec{\ell}}$ are positive. Thus $\boldsymbol{K}_{\vec{k}}$ and $\boldsymbol{K}_{\vec{\ell}}$ are negative definite matrices.

- strongly future trapped if $\vec{G}$ is past-pointing on $S$; equivalently, $\operatorname{det} \boldsymbol{K}_{\vec{k}}$ and $\operatorname{det} \boldsymbol{K}_{\vec{\ell}}$ are non-negative, so that $\boldsymbol{K}_{\vec{k}}$ and $\boldsymbol{K}_{\vec{\ell}}$ are negative semi-definite. If in addition $\vec{G}$ is nonzero on $S$, then $S$ is called very strongly future trapped. In this case, $\operatorname{det} \boldsymbol{K}_{\vec{k}}$ and $\operatorname{det} \boldsymbol{K}_{\vec{\ell}}$ do not vanish simultaneously.

Similarly, a marginally future-trapped surface (so that $\vec{H} \neq \overrightarrow{0}$ is null and future pointing) is said to be:

- totally marginally future-trapped if $\vec{G}$ is past null (necessarily) collinear with $\vec{H}$; equivalently, one of $\operatorname{det} \boldsymbol{K}_{\vec{k}}$ or $\operatorname{det} \boldsymbol{K}_{\vec{\ell}}$ vanishes and the other is positive. Hence, one of $\boldsymbol{K}_{\vec{k}}$ or $\boldsymbol{K}_{\vec{\ell}}$ vanishes and the other is negative definite.

- very strongly marginally future-trapped if $\vec{G}$ is past causal (necessarily collinear with $\vec{H}$ ): one of $\operatorname{det} \boldsymbol{K}_{\vec{k}}$ or $\operatorname{det} \boldsymbol{K}_{\vec{\ell}}$ vanishes, the other is non-negative. Hence, one of $\boldsymbol{K}_{\vec{k}}$ or $\boldsymbol{K}_{\vec{\ell}}$ vanishes, the other is negative semi-definite.

Strongly or higher (marginally) trapped surfaces are such that the norms of all their tangent vectors are nonincreasing along any orthogonal future direction. Some examples of these surfaces in well-known black hole solutions are mentioned below — after Result 1 -, see also [62].

Now, let $\mathcal{C}$ be a differentiable curve in $S$. Regarded as a curve in the whole $\mathcal{V}, \mathcal{C} \subset S$ is obviously a spacelike curve. Let $\vec{v} \in \mathfrak{X}(\mathcal{C}) \subset \mathfrak{X}(S)$ be the unit tangent vector to $\mathcal{C}$, then the acceleration (or curvature) of $\mathcal{C}$ in $\mathcal{V}$ is

$$
\nabla_{\vec{v}} \vec{v}=-\vec{\kappa}(\vec{v})-\vec{K}(\vec{v}, \vec{v}) \equiv-\vec{\varkappa}(\vec{v}) .
$$

Here $\vec{\kappa}(\vec{v}) \in \mathfrak{X}(\mathcal{C})^{\perp} \cap \mathfrak{X}(S)$ is the acceleration of $\mathcal{C}$ as a curve in $(S, \gamma)$, and $\vec{\varkappa}(\vec{v}) \in \mathfrak{X}(\mathcal{C})^{\perp} \cdot \vec{\kappa}(\vec{v})$ and $\vec{\varkappa}(\vec{v})$ are actually the mean curvature vectors of $\mathcal{C}$ in $S$ and $\mathcal{V}$, respectively. 
$\mathcal{C}$ is therefore (marginally) future trapped whenever $\vec{\varkappa}(\vec{v})$ is (null) timelike and future pointing [48].

Take now two curves $\mathcal{C}_{1}$ and $\mathcal{C}_{2}$ in $S$, and assume that they meet orthogonally at a point $x \in \mathcal{C}_{1} \cap \mathcal{C}_{2} \subset S$. Using (4) for both curves we obtain

$$
\left.\vec{H}\right|_{x}=\left.\left(\vec{\varkappa}\left(\vec{v}_{1}\right)-\vec{\kappa}\left(\vec{v}_{1}\right)\right)\right|_{x}+\left.\left(\vec{\varkappa}\left(\vec{v}_{2}\right)-\vec{\kappa}\left(\vec{v}_{2}\right)\right)\right|_{x} .
$$

Since $\vec{H}$ is orthogonal to both $\vec{\kappa}\left(\vec{v}_{1}\right) \propto \vec{v}_{2}$ and $\vec{\kappa}\left(\vec{v}_{2}\right) \propto \vec{v}_{1}$, $\vec{H}$ is future pointing whenever so are $\vec{\varkappa}\left(\vec{v}_{1}\right)$ and $\vec{\varkappa}\left(\vec{v}_{2}\right)$. However, this is too strong a requirement, as it is clear from (5): it is sufficient that $\vec{\varkappa}\left(\vec{v}_{1}\right)-\vec{\kappa}\left(\vec{v}_{1}\right)$ and $\vec{\varkappa}\left(\vec{v}_{2}\right)-$ $\vec{\kappa}\left(\vec{v}_{2}\right)$ be future-pointing to get the same conclusion. Noting that $\vec{\varkappa}(\vec{v})-\vec{\kappa}(\vec{v})=\overrightarrow{\boldsymbol{K}}(\vec{v}, \vec{v}) \in \mathfrak{X}(S)^{\perp}$ is the projection orthogonal to $S$ of the mean curvature vector of $\mathcal{C}$ in $\mathcal{V}$, the following definition is in order: any $\mathcal{C} \subset S$ with a future-pointing (null) timelike $\vec{\varkappa}(\vec{v})-\vec{\kappa}(\vec{v})$ will be called $S$-outwardly (marginally) future trapped.

Thus, the following elementary result holds:

If two $S$-outwardly future trapped curves in $S$ meet orthogonally at $x \in S$, then $S$ is contracting at $x$. While if two $S$-outwardly marginally future trapped curves in $S$ are orthogonal at $x \in S$, then $S$ is either semi-contracting or contracting at $x$ depending on whether $\vec{\varkappa}\left(\vec{v}_{1}\right)-\vec{\kappa}\left(\vec{v}_{1}\right)$ and $\vec{\varkappa}\left(\vec{v}_{2}\right)-\vec{\kappa}\left(\vec{v}_{2}\right)$ are collinear at $x$ or not. (If they are collinear, they are called consistently marginally trapped.)

Remarks: (1) The first conclusion is stronger than stated: not only $S$ is contracting $\left(\left.\theta_{\vec{k}}\right|_{x}<0\right.$ and $\left.\left.\theta_{\vec{\ell}}\right|_{x}<0\right)$, also $\left.\boldsymbol{K}_{\vec{k}}\left(\vec{v}_{i}, \vec{v}_{i}\right)\right|_{x}<0$ and $\left.\boldsymbol{K}_{\vec{\ell}}\left(\vec{v}_{i}, \vec{v}_{i}\right)\right|_{x}<0$ for $i=1,2$. This implies that $\left.\overrightarrow{\boldsymbol{K}}\left(\vec{v}_{i}, \vec{v}_{i}\right)\right|_{x}$ are future timelike for both orthogonal directions $\vec{v}_{i}$. This can happen only for some types of contracting points (Appendix in [62]). (2) If every curve tangent to $S$ at $x \in S$ is $S$-outwardly future trapped, then $\left.\boldsymbol{K}_{\vec{k}}\right|_{x}$ and $\left.\boldsymbol{K}_{\vec{\ell}}\right|_{x}$ are negative definite matrices.

The previous elementary result is local and independent of the topology of $S$. It also applies to planes $\left(\mathbb{R}^{2}\right)$ or cylinders $\left(S^{1} \times \mathbb{R}\right)$, so any hoop idea is yet missing. Recall that there are non-compact trapped surfaces with those topologies in flat space-time (e.g. Example 4.1 in [61], p.776), and thus they cannot be related to horizons or black holes.

To link trapped circles with the Hoop Conjecture one has to deal with compact $S$ and use closed loops $\mathcal{C}$ that "go round $S$ " —not just local loops near $x$. One possibility is to use, for each $x \in S$, congruences of loops emanating from $x$ which cover the entire $S$ [26], then selecting their members not contained in any convex neighborhood (in $S$ ). The required hoops will be those with greater lengths on each possible family. Another possibility would be to employ closed geodesics in $S$. Periodic geodesics intuitively seem to do the job (e.g. in the standard round sphere they are the great circles $\left.2^{2}\right)$. It is interesting to

${ }^{2}$ However, in the standard torus $S^{1} \times S^{1}$ all its meridians are closed geodesics but, letting aside the inner and outer equators, their corresponding orthogonal closed geodesics go "up and down" crossing the outer equator. To use them as good models of a hoop is debatable. Nonetheless, another simpler possibility in this case is to use the topological foliation defined by a set of meridians and their everywhere orthogonal circles. remark that $\vec{\kappa}(\vec{v})=\overrightarrow{0}$ for geodesics, hence $S$-outwardly trapped geodesics are simply trapped. Closed geodesics in relation with the Hoop Conjecture were used in [19,21]. Unfortunately, their existence for arbitrary $x \in S$ is not guaranteed for the important case of a topological sphere 3

I will use the term hoop for the appropriate closed loops "going round" $S$, and I take their abundance for granted. These are not rigid hoops, rather they fit the surface, adapting themselves in order to conform with the shape of $S$. Applying the previous results to any closed spacelike surface $S$, the following preliminary result holds

Proposition 1 If two (consistently marginally) $S$ outwardly future-trapped orthogonal hoops pass through every $x \in S$, then $S$ is (marginally) future trapped.

If all hoops are $S$-outwardly (marginally) future trapped, then $S$ is totally (marginally) future trapped.

The converse of the last statement in this proposition also holds. For any two unit orthogonal vectors $\vec{v}_{1}, \vec{v}_{2} \in$ $T_{x} S$, two curves tangent to $\vec{v}_{1}$ and $\vec{v}_{2}$ will have futurepointing $\vec{\varkappa}\left(\vec{v}_{1}\right)-\left.\vec{\kappa}\left(\vec{v}_{1}\right)\right|_{x}$ and $\vec{\varkappa}\left(\vec{v}_{2}\right)-\left.\vec{\kappa}\left(\vec{v}_{2}\right)\right|_{x}$ if

$$
\boldsymbol{K}_{\vec{k}}\left(\vec{v}_{i}, \vec{v}_{i}\right) \leq 0, \quad \boldsymbol{K}_{\vec{\ell}}\left(\vec{v}_{i}, \vec{v}_{i}\right) \leq 0 \quad \text { for } i=1,2 .
$$

For totally (marginally) future trapped surfaces, these conditions are trivially met for all vectors $\vec{v} \in T_{x} S$ ergo:

Proposition 2 If $S$ is a closed totally (marginally) future-trapped surface, all possible hoops through all $x \in S$ are $S$-outwardly (consistently marginally) future trapped.

Actually, the "total" condition can be relaxed. For (very) strongly future-trapped surfaces, $\overrightarrow{\boldsymbol{K}}(\vec{v}, \vec{v})$ is timelike and future pointing for all $\vec{v} \in T_{x} S$ except for, at most, (one) two directions at each $x \in S$ [62]. Analogously, if $S$ is very strongly marginally future trapped, then $\overrightarrow{\boldsymbol{K}}(\vec{v}, \vec{v})$ are consistently null and future pointing for all $\vec{v} \in T_{x} S$ except for, at most, one direction at each $x \in S$. In both situations it is obvious that almost all possible hoops are $S$-outwardly (marginally) future-trapped. Observe that if a hoop tends to be tangent to any of the exceptional tangent vectors, it is enough to slightly modify the hoop, keeping its essential properties, to avoid this tangency. Thus

Proposition 3 If $S$ is a closed strongly future-trapped surface, almost every pair of mutually orthogonal hoops through every $x \in S$ is $S$-outwardly future trapped.

If $S$ is a closed very strongly marginally futuretrapped surface, almost every pair of mutually orthogonal hoops through every $x \in S$ is $S$-outwardly consistently marginally future trapped.

\footnotetext{
${ }^{3}$ For general closed surfaces $S$ with any smooth Riemannian metric, any non-trivial free homotopy class has a closed geodesic, from which there are in fact infinitely many closed geodesics for non- $S^{2}$ surfaces [41]. The problematic case is that of surfaces of spherical topology $S^{2}$-the most important case due to the topological censorship results $[17,31,32,35]$. It is now known $[5,27,37]$ that independently of the Riemannian metric equipping $S^{2}$, there always exist an infinite number of closed geodesics. Unfortunately, one does not know how many of them pass through a particular given point.
} 
A strict converse of the first statement in Proposition 1 encounters some technical difficulties, as conditions (6) may not hold for general future-trapped surfaces. The problem is that a future-pointing $\vec{H}$ does not imply, in general, that both $\vec{\varkappa}\left(\vec{v}_{1}\right)-\vec{\kappa}\left(\vec{v}_{1}\right)$ and $\vec{\varkappa}\left(\vec{v}_{2}\right)-\vec{\kappa}\left(\vec{v}_{2}\right)$ are future-pointing. It is easy to prove, though, that (6) do hold at least for one of the two null second fundamental forms by choosing the $\vec{v}_{i}$ appropriately [62]: diagonalize (say) $\left.\boldsymbol{K}_{\vec{k}}\right|_{x}$, so that if $\vec{e}_{1}, \vec{e}_{2} \in T_{x} S$ are its unit eigenvectors and $\lambda_{1}, \lambda_{2}$ the corresponding eigenvalues, then $\left.\theta_{\vec{k}}\right|_{x}=\lambda_{1}+$ $\lambda_{2} \leq 0$. Defining two new orthogonal unit vectors by

$$
\left(\begin{array}{l}
\vec{v}_{1} \\
\vec{v}_{2}
\end{array}\right)=\left(\begin{array}{cc}
\cos \beta & -\sin \beta \\
\sin \beta & \cos \beta
\end{array}\right)\left(\begin{array}{l}
\vec{e}_{1} \\
\vec{e}_{2}
\end{array}\right)
$$

one gets $-\boldsymbol{K}_{\vec{k}}\left(\vec{v}_{1}, \vec{v}_{1}\right)=-\lambda_{1} \cos ^{2} \beta-\lambda_{2} \sin ^{2} \beta$, $-\boldsymbol{K}_{\vec{k}}\left(\vec{v}_{2}, \vec{v}_{2}\right)=-\lambda_{2} \cos ^{2} \beta-\lambda_{1} \sin ^{2} \beta$ which will be both non-negative for at least the value $\beta=\pi / 4$. The same can be proven for $\boldsymbol{K}_{\vec{\ell}}$, leading to another set of pairs of orthonormal vectors $\vec{v}_{1}^{\prime}, \vec{v}_{2}^{\prime} \in T_{x} S$. But, if the sets of allowed directions $\vec{v}_{1}, \vec{v}_{2}$ for $\boldsymbol{K}_{\vec{k}}$ and $\vec{v}_{1}^{\prime}, \vec{v}_{2}^{\prime}$ for $\boldsymbol{K}_{\vec{\ell}}$ do not have pairs in common, then conditions (6) will not hold simultaneously, and therefore there will be $x \in S$ such that no pair of $S$-outwardly future-trapped curves is orthogonal at $x$.

To overcome these impediments one needs the existence of very few directions $\vec{v}$ for which $\overrightarrow{\boldsymbol{K}}(\vec{v}, \vec{v})$ is not future pointing. A good possibility to achieve this consists in restricting oneself to very lightly (strongly marginally) future trapped surfaces [62], characterized by having one of the null second fundamental forms negative semi-definite (vanishing). If $\boldsymbol{K}_{\vec{k}}$ is not the semi-definite (vanishing) matrix, it all depends on the magnitude at each point of the angle

$$
\arcsin \sqrt{\lambda_{1} /\left(\lambda_{1}-\lambda_{2}\right)}
$$

(or the corresponding angle for $\boldsymbol{K}_{\vec{\ell}}$ in the other case) where $\lambda_{1}$ is the negative eigenvalue and $\left|\lambda_{1}\right|>\lambda_{2}>0$. Let us call this value the critical angle. The larger this angle, the better. Thus, as a complement to Propositions 2 and 3 there is a stronger result of the following kind:

Result 1 If the critical angle of a closed very lightly (strongly marginally) future-trapped surface $S$ is bounded below by a value near enough $\pi / 2$, then two mutually orthogonal (consistently marginally) S-outwardly futuretrapped hoops pass through every $x \in S$.

Propositions 1, 2, 3 and Result 11 provide if-and-only-if formulations of (a) the notion "severely compacted in every direction" and (b) how to superpose the hoops avoiding any slicing. The pairs of mutually orthogonal and $S$-outwardly (marginally) future-trapped hoops build up a "cage" confining $S$. For strongly or totally trapped $S$, they actually add up to a perfectly fitting adjustable dress.

An open question is the exact lower bound for the critical angle defining the sharpest combination of Result 1 and the first statement in Proposition 1 Nevertheless, this might turn out to be irrelevant if we accept that, in realistic collapses, dynamical/trapping horizons in black hole spacetimes will eventually approach the event horizon and, therefore, be foliated by very strongly marginally trapped surfaces. This is supported by several results: (i) the probable eventual mergence of event horizons and hypersurfaces foliated by marginally trapped closed surfaces $[2,15]$, discussed in [40] and partly confirmed under some circumstances or numerically, e.g. $[2-4,14,69]$ and references therein; (ii) the black hole "rigidity" $[22,32]$ and uniqueness theorems, e.g. $[36,58]$ and their references, the former stating that event horizons of stationary black holes are Killing horizons, the latter that electrovacuum black holes in equilibrium are of Kerr-Newman type; (iii) the fact that non-expanding horizons, including isolated and Killing horizons, are foliated by strongly (or higher) marginally trapped surfaces $[3,29]$; in particular the KerrNewman event horizon is foliated by totally marginally trapped surfaces. Observe that tubes foliated by strictly stable [1] marginally trapped surfaces are completely partitioned into isolated and dynamical horizons $[1,16]$; (iv) the properties of spherically symmetric horizons, necessarily foliated by totally marginally future-trapped spheres [62]

Taking into account that dynamical horizons have a unique foliation by marginally trapped surfaces [2], I would like to put forward a new conjecture, advanced in [62], concerning the "peeling behavior" of these horizons: their closed marginally future-trapped surfaces become increasingly trapped and eventually are very strongly future trapped, whenever the physical system is realistic and leads to the formation of a black hole in equilibrium.

Propositions 1, 2, 3 and Result 1 are purely geometrical results, independent of energy conditions or field equations. These are however fundamental for singularity theorems $[32,33,53,61]$ and for the issues mentioned in the previous two paragraphs. Hence, they are needed to promote the results in this paper into a physical statement, and for the previous conjecture. As the results only resolve geometrical problems of the Hoop Conjecture, one main ingredient is still missing: the relation between geometry and matter, or how to produce a formula relating a length with the mass of a black hole once energy conditions have been assumed. This problem can be addressed: an appropriate length can be defined, in the frame of the reformulation, by considering all possible $S$-outwardly marginally futuretrapped hoops in a marginally future-trapped $S$ such that the projection orthogonal to $S$ of their mean curvature vector is parallel to $\vec{H}$ (consistency). For each $x \in S$, take the infimum of the lengths of all such hoops $\mathcal{H}_{x}$ from $x$ and denote it by $D\left(\mathcal{H}_{x}\right)$. Now, simply put by definition

$$
C \equiv \sup _{x \in S} D\left(\mathcal{H}_{x}\right)
$$

which is generically smaller than twice the diameter of $S$

${ }^{4}$ This is reasonable only for $S^{2}$ topology. Otherwise, each nontrivial free homotopy class has a length $C_{k}$ : the supremum for $x \in S$ of the infima of the lengths of $\mathcal{H}_{x}$ in that class. The number of $C_{k}$ depends on the genus of $S$. However, finding a geometric measure of enclosed mass is also problematic in these cases. 
(the supremum of distances between pairs of points).

There only remains to find an appropriate measure for the "mass". This can be estimated by means of any of the available quasi-local masses [64]. A fundamental requirement for all of them is that marginally trapped surfaces with $S^{2}$-topology enclose a quasi-local mass equal to its so-called irreducible mass ( [64], sect. 4.3.2, 1.7), that is

$$
M_{i r r}=\left(c^{2} / 4 G\right) \sqrt{\operatorname{Area}(S) / \pi} .
$$

One may be tempted to write now the formula

$$
C \leq \sqrt{\pi \operatorname{Area}(S)}
$$

so that this coincides, via (7), with the original Hoop formulation where the mass is $M_{i r r}$. This formula appears to be true in static cases. Unfortunately, it is a matter of simple calculation to check that (8) fails for Kerr black holes near the extreme case. Hence, we need a better estimate of the right-hand side of the inequality (8).

In non-static cases the main new, and fundamental, ingredient is rotation, therefore angular momentum has to be taken into consideration. It is well-known, e.g. $[3,29,30,56]$, that angular momentum is related to the one-form $\boldsymbol{\theta} \in \Lambda^{1} S$ defined as $\boldsymbol{\theta}(\vec{X}) \equiv g\left(\vec{\ell}, \nabla_{\vec{X}} \vec{k}\right)$ for every $\vec{X} \in \mathfrak{X}(S)$. This one-form is a connection on the normal bundle of $S$, and its change under (10) is [62] $\boldsymbol{\theta}^{\prime}=\boldsymbol{\theta}+d \log \sigma^{2}$. Thus, its curvature $d \boldsymbol{\theta} \in \Lambda^{2} S$ is invariant under (10). The standard way to define angular momentum is to consider a fixed rotational vector field $\vec{\xi} \in \mathfrak{X}(S)$ and integrating $\boldsymbol{\theta}(\vec{\xi})$ on the compact $S$. This, of course, depends on the representative chosen for $\vec{\ell}[3]$, so it is not invariant under (11) - unless $\vec{\xi}$ is divergencefree. Moreover, this requires a preferred rotational $\vec{\xi}$ on $S$. These properties are not desirable for the geometric formulation that I have in mind.

However, the invariant 2-form $d \boldsymbol{\theta}$ can be used for our purposes. As a matter of fact, this 2-form is known to be related to angular momentum and gravitational radiation, see [25], which is the second new ingredient in non-static situations. First, define its magnitude as usual: $\mathcal{R}^{2} \equiv(1 / 2) \boldsymbol{\gamma}(d \boldsymbol{\theta}, d \boldsymbol{\theta})=2 \theta_{[A ; B]} \theta^{[A ; B]} \geq 0$. Normalize it with respect to the curvature on $S$ dividing by $Q \equiv \sup _{x \in S} K_{S}>0$ where $K_{S}$ is the Gaussian curvature of $S$. Integrate this over the compact $S$ to obtain the quantity

$$
\operatorname{Rot}(S) \equiv \frac{1}{Q} \int_{S} \mathcal{R}
$$

With these well-posed definitions for $C$ and $\operatorname{Rot}(S)$, a geometric Hoop formula can be explicitly written as

$$
C \leq \sqrt{\pi[\operatorname{Area}(S)+b \operatorname{Rot}(S)]}
$$

where $b$ is a positive number. It is feasible in principle that this inequality held for untrapped surfaces (if the angular momentum is very large for a given mass), so that for the "only when" part of the conjecture $S$ in (9) should probably be understood as (approaching) a very strongly marginally future-trapped $S^{2}$-surface so that the "peeling off" has taken place and the critical angle is large enough. In order to fix $b$ one can use the standard Kerr black holes. An elementary calculation proves that the above inequality is saturated for the cases of (i) the Schwarzschild black hole $(\operatorname{Rot}(S)=0)$ and (ii) the extreme Kerr solution, in both cases exactly for the value

$$
b=2 .
$$

For this value, the inequality is strict in any other Kerr black hole. One wonders whether the right-hand side of the inequality (9) may lead to a new definition of "mass", but this is outside the scope of this letter.

Let me finally consider whether the reformulation can be extended to dimensions $n \geq 4$. By carefully dissecting the reasonings used to prove the results one realizes that higher dimensions request families of closed $(n-3)$ dimensional submanifolds, in agreement with [6,38,72]. The generalization of the basic relation (5) to higher values of $n$ is then straightforward. Take $n-2$ hypersurfaces $\mathcal{C}_{j} \subset S$ of a co-dimension 2 embedded submanifold $S$ such that they meet mutually orthogonally at $x \in S$. Then

$$
\left.\vec{H}\right|_{x}=\left.\frac{1}{n-3} \sum_{j=1}^{n-2}\left(\vec{\varkappa}_{j}-\vec{\kappa}_{j}\right)\right|_{x}
$$

where $\vec{\varkappa}_{j}$ and $\vec{\kappa}_{j}$ are the mean curvature vectors of each $\mathcal{C}_{j}$ in $\mathcal{V}$ and in $S$ respectively. However, the election of the "hoops" $\mathcal{C}_{j}$ must be done with care depending on the topology of $S$, see $[17,35]$. For $S^{n-2}$-topology one has to choose $(n-3)$-dimensional spheres, but for a black ring [24] with topology $S^{1} \times S^{n-3}$ the choice is one hoop with topology $S^{n-3}$ and $n-3$ hoops with topology $S^{1} \times S^{n-4}$.

Other difficulties may arise due to the peculiar properties of spherical black holes in higher dimensions. For instance, in dimensions $n>5$ the horizon may have very different sizes in different directions, with arbitrarily large angular momentum for a given mass resulting in a tiny $(n-2)$-volume for $S$ [23]. However, this volume scales as $M r_{+}$, where $M$ is the mass and $r_{+}$is typically of the order of the horizon's small characteristic length, ergo an inequality of the type shown in this paper might still be possible for such ultra-spinning black holes.

$$
* * *
$$

I am indebted to R Emparan, E Malec, M Mars, M Sánchez and R Vera for many suggestions. I acknowledge support from grants FIS2004-01626 and GIU06/37.

\section{REFERENCES}

[1] L. Andersson, M. Mars, and W. Simon, Phys. Rev. Lett., 95 (2005) 111102

[2] A. Ashtekar and G.J. Galloway, Adv. Theor. Math. Phys., 9 (2005) 1 
[3] A. Ashtekar and B. Krishnan, Phys. Rev. Lett., 89 (2002) 261101; Living Rev. Relativity, 7 (2004) 10

[4] L. Baiotti, I. Hawke, P.J. Montero, F. Loeffler, L. Rezzolla, N. Stergioulas, J.A. Font, and E. Seidel, Phys. Rev. D, 71 (2005) 024035

[5] V. Bangert, Internat. J. Math., 4 (1993) 1

[6] C. Barrabès, V.P. Frolov, and E. Lesigne, Phys. Rev. D, 69 (2004) 101501

[7] C. Barrabès, W. Israel, and P. Letelier, Phys. Lett. A, 160 (1991) 41

[8] P. Bizon and E. Malec, Phys. Rev. D, 40 (1989) 2559

[9] P. Bizon, E. Malec, and N. O'Murchadha, Phys. Rev. Lett., 61 (1988) 1147

[10] P. Bizon, E. Malec, and N. O’Murchadha, Class. Quantum Grav., 6 (1989) 961

[11] P. Bizon, E. Malec, and N. O’Murchadha, Class. Quantum Grav., 7 (1990) 1953

[12] W.B. Bonnor, Phys. Lett. A, 99 (1983) 424

[13] W.B. Bonnor, Class. Quantum Grav., 15 (1998) 351

[14] I. Воотн, Can. J. Phys., 83 (2005) 1073

[15] I. Booth, L. Brits, J.A. GonzÁlez and C. VAn den Broeck, Class. Quantum Grav., 23 (2006) 413

[16] I. Booth and S. Fairhurst, Phys. Rev. D, 75 (2007) 084019

[17] M. Cai and G.J. Galloway, Class. Quantum Grav., 18 (2001) 2707

[18] A. Chamorro, R. Gregory, and J.M. Stewart, Proc. R. Soc. London A, 413 (1987) 251

[19] T. Chiba, Phys. Rev. D, 60 (1999) 044003

[20] T. Chiba and K. Maeda, Phys. Rev. D, 50 (1994) 4903

[21] T. Chiba, T. Nakamura, K. Nakao, and M. Sasaki, Class. Quantum Grav., 11 (1994) 431

[22] P. Chruściel, Commun. Math. Phys., 189 (1997) 1

[23] R. Emparan and R.C. Myers, JHEP, 09 (2003) 025

[24] R. Emparan and H.S. Reall, Phys. Rev. Lett., 88 (2002) 101101; Class. Quantum Grav., 23 (2006) R169

[25] R.J. Epp, Phys. Rev. D, 62 (2000) 124018

[26] E. Flanagan, Phys. Rev. D, 44 (1991) 2409; Phys. Rev. D, 46 (1429) 1992

[27] J. FrAnKs, Invent. Math., 108 (1992) 403

[28] G.W. Gibbons, Commun. Math. Phys., 27 (1972) 87; in Global Riemannian Geometry, edited by T.J. Willmore and N.J. Hitchin (Ellis Harwood, Chichester) 1984, p. 194

[29] E. Gourgoulhon and J.L. Jaramillo, Phys. Rep., 423 (2006) 159

[30] P. Hajicek, J. Math. Phys., 15 (1974) 1554; J. Math. Phys., 16 (1975) 518; J. Math. Phys., 16 (1975) 523

[31] S.W. Hawking, Commun. Math. Phys., 25 (1972) 152

[32] S.W. Hawking and G.F.R. Ellis, The Large Scale Structure of Space-Time (Cambridge Univ. Press, Cambridge) 1973

[33] S.W. Hawking and R. Penrose, Proc. Roy. Soc. London A, 314 (1970) 529

[34] S.A. Hayward, Phys. Rev. D, 49 (1994) 6467; Class. Quantum Grav., 11 (1994) 3025

[35] C. Helfgott, Y. Oz, and Y. Yanay, JHEP, 0602 (2006) 025

[36] M. Heusler, Black Hole Uniqueness Theorems (Cambridge Univ. Press, Cambridge) 1996

[37] N. Hingston, Internat. Math. Res. Notices, 9 (1993) 253

[38] D. Ida And K. Nakao, Phys. Rev. D, 66 (2002) 064026
[39] D. Ida, K. Nakao, M. Silno, and S.A. Hayward, Phys. Rev. D, 58 (1998) 121501

[40] W. Israel, Can. J. Phys., 64 (1986) 120; Phys. Rev. Lett., 56 (1986) 789

[41] W. KlingenBerG, Lectures on closed geodesics. Grundlehren d. Math. Wiss. (Springer, Berlin), Vol. 230 1978

[42] M. KRIELE, Spacetime (Springer, Berlin) 1999

[43] M. Kriele and S. A. Hayward, J. Math. Phys., 38 (1997) 1593

[44] D.M. Kupeli, Gen. Rel. Grav., 19 (1987) 23

[45] J.P.S. Lemos, Phys. Rev. D, 59 (1999) 044020

[46] E. Malec, Phys. Rev. Lett., 67 (1991) 949; Acta Phys. Pol. B, 22 (1991) 829

[47] E. Malec and P. Koc, Acta Phys. Pol. B, 23 (1992) 123

[48] M. Mars and J.M.M. Senovilla, Class. Quantum Grav., 20 (2003) L293

[49] C. W. Misner, K.S. Thorne, and J.A. Wheeler, Gravitation, (W.H. Freeman and Co., New York) 1973

[50] T. Nakamura, S.L. Shapiro, and S.A. Teukolsky, Phys. Rev. D, 38 (1988) 2972

[51] B. O'NeILl, Semi-Riemannian Geometry (Academic P., New York) 1983

[52] M.A. Pelath, K.P. Tod, and R.M. Wald, Class. Quantum Grav., 15 (1998) 3917

[53] R. Penrose, Phys. Rev. Lett., 14 (1965) 57

[54] R. Penrose, Riv. Nuovo Cimento, 1 (1969) 252

[55] R. Penrose, Ann. N.Y. Acad. Sci., 224 (1973) 125

[56] R.H. Price and K.S. Thorne, Phys. Rev. D, 33 (1986) 915

[57] I.H. Redmount, Phys. Rev. D, 27 (1983) 699

[58] D.C. Robinson, "Four decades of black hole uniqueness theorems", To appear in: The Kerr Space-time: Rotating Black Holes in General Relativity, edited by D.L. WILTSHIRE, M. Visser and S.M. ScotT (Cambridge University Press, Cambridge) 2007

[59] R. Schoen and S.T. Yau, Commun. Math. Phys., 90 (1983) 575

[60] J. Seifert, Gen. Rel. Grav., 10 (1979) 1065

[61] J.M.M. Senovilla, Gen. Rel. Grav., 30 (1998) 701

[62] J.M.M. Senovilla, Class. Quantum Grav., 24 (2007) 3091

[63] S.L. Shapiro and S.A. Teukolsky, Phys. Rev. Lett., 66 (1991) 994

[64] L. Szabados, Living Rev. Relativity, 7 (2004) 4

[65] K.S. Thorne, in Magic without Magic: John Archibald Wheeler, edited by J. KLAuder (Freeman, S. Francisco) 1972, p. 231

[66] K.P. Tod, Class. Quantum Grav., 9 (1992) 1581

[67] R.M. WALD, "Gravitational Collapse and Cosmic Censorship" in Black Holes, Gravitational Radiation and the Universe, edited by B.R. IYER and B. BHAWAL (Springer, Berlin) 1998

[68] R.M. Wald and V. Iyer, Phys. Rev. D, 44 (1991) R3719

[69] C. Williams, "Asymptotic Behavior of Spherically Symmetric Marginally Trapped Tubes" arXiv:gr-qc/0702101

[70] J. Wojtkiewicz, Phys. Rev. D, 41 (1990) 1867

[71] J. Wojtkiewicz, Class. Quantum Grav., 9 (1992) 1255

[72] H. Yoshino and Y. NAmbu, Phys. Rev. D, 66 (2002) 065004

[73] H. Yoshino, Y. Nambu, and A. Tomimatsu, Phys. Rev. $D, 65$ (2002) 064034 